\title{
O CONHECIMENTO DE FUTUROS PROFESSORES DE MATEMÁTICA SOBRE O CONCEITO DE FUNÇÃO E POSSÍVEIS IMPLICAÇÕES PARA A PRÁTICA DOCENTE
}

\author{
THE KNOWLEDGE OF THE FUTURE MATHEMATICS TEACHERS ABOUT \\ FUNCTION CONCEPT E POSSIBLE IMPLICATIONS FOR THE TEACHERS \\ PRACTICE
}

\author{
Fabíola Peixoto Cintra \\ Rede Estadual de São Paulo \\ fabiolacintra17@hotmail.com \\ Inocêncio Fernandes Balieiro Filho \\ Universidade Estadual Paulista - UNESP \\ inocencio.balieiro@unesp.br
}

\begin{abstract}
Resumo
O conhecimento dos professores, juntamente com as suas concepções e crenças, é uma importante variável no processo de ensino e de aprendizagem da Matemática que ocorre em suas aulas, uma vez que o conhecimento do professor é a base para o ensino de um determinado conteúdo. Nesta perspectiva, o objetivo da pesquisa aqui apresentada foi investigar os conhecimentos dos futuros professores de Matemática sobre o conceito de Função e apontar suas possíveis implicações para a atividade docente sobre o ensino e a aprendizagem desse conceito. Para isso, tomando como base uma revisão histórica do desenvolvimento do conceito de função e uma revisão bibliográfica sobre o conhecimento dos professores sobre esse conceito, foi elaborado e aplicado um questionário para alunos do último ano de um curso de Licenciatura em Matemática. Os resultados obtidos revelam que, de modo geral, o conceito de função está sendo adequadamente estudado e aprendido por esses alunos, mas ainda existem obstáculos epistemológicos que necessitam ser superados para uma compreensão abrangente do conceito de função, ou seja, há algumas dificuldades de aprendizagem dos alunos em relação ao conceito de função, em virtude da sua variedade de representações, levando aos obstáculos epistemológicos.

Palavras-chave: Conceito de função. Formação inicial de professores. Conhecimento e prática docente.
\end{abstract}

\begin{abstract}
Teachers' knowledge, together with their conceptions and beliefs, is an important variable in the teaching and learning process of Mathematics that occurs in their classes, since the knowledge of
\end{abstract}

ReviSeM, Ano 2019, N. 2, p. $48-70$ 48 
the teacher is the basis for the teaching of a certain content. In this perspective, the purpose of the research presented here was to investigate the knowledge of the future Mathematics teachers about the concept of Function and point out their possible implications for the teachers practice about the teaching and learning of this concept. Based on a historical review of the development of the concept of function and a bibliographical revision on the teachers' knowledge about this concept, a questionnaire was elaborated and applied to students of the last year of a degree in Mathematics and a questionnaire was sent to the teachers of this course. The results show that function concept is be studied and learned by students, but there are still has epistemological obstacles that need to be overcome for a embracing function concept understanding, because of your representations variety, which leads to epistemological obstacles.

Keywords: Concept of function. Initial teacher training. Knowledge and teaching practice.

\section{INTRODUÇÃO}

O conceito de função, até o século XX, passou por diversos obstáculos: problemas relacionados à utilização do conceito na prática, problemas numéricos, algébricos e geométricos (SIERPINSKA, 1992). Dessa forma, é compreensível que os alunos dos diferentes níveis de ensino tenham dificuldades na compreensão do conceito de função.

Diversos estudos, conforme citado em Llinares (1996), apontam que o conhecimento dos professores, juntamente com as suas concepções e crenças, é uma importante variável do processo de ensino e de aprendizagem da Matemática que ocorre em suas aulas, uma vez que o conhecimento do professor é a base para o ensino de um determinado conteúdo. Além disso, o conhecimento matemático do professor tem relações com suas crenças e concepções, com o conhecimento pedagógico do conteúdo e com sua prática.

Shulman (1986) sugere que o conhecimento do professor é ramificado e composto tanto pelo conhecimento do conteúdo, como pelo conhecimento didático do conteúdo e, tal conhecimento (Pedagogical Content Knowledge) envolve:

Dentro da categoria de conhecimento pedagógico do conteúdo, incluo, para os assuntos mais frequentemente ensinados em determinadas áreas do conhecimento, as formas mais úteis de representação dessas ideias, as analogias mais poderosas, ilustrações, exemplos e demonstrações em resumo, as formas de representar e formular o assunto de modo que o torne compreensível aos demais. Uma vez que não existem formas mais poderosas de representação, o professor tem de ter em mãos um verdadeiro arsenal de formas alternativas de representação, com algumas que derivam da pesquisa e outras que têm sua origem no saber da prática. (SHULMAN, 1986, p. 9).

ReviSeM, Ano 2019, N. 2, p. $48-70$ 
Desse modo, o conhecimento pedagógico do conteúdo é uma categoria específica do conhecimento dentro da prática, ou seja, Shulman valoriza o conteúdo em si, sem deixar de lado o pedagógico. Portanto, o conhecimento pedagógico do conteúdo é uma junção do conteúdo e da pedagogia, o qual permite que o professor tenha a capacidade de transformar o conteúdo por meio das abordagens pedagógicas e adaptá-las para a aprendizagem do aluno.

Nesta perspectiva, neste artigo, temos como intuito investigar os conhecimentos dos futuros professores de Matemática sobre um dos conceitos que fazem parte da base dos Fundamentos da Matemática, o conceito de Função, e apontar suas possíveis implicações para a atividade docente sobre o ensino e a aprendizagem desse conceito.

\section{CONHECIMENTO DO PROFESSOR DE MATEMÁTICA SOBRE O CONCEITO DE FUNÇÃO}

O conceito de função é fundamental para a organização dos ramos e dos conhecimentos específicos da Matemática, desempenhando também um papel importante em outras áreas do conhecimento, em virtude de sua aplicabilidade. Assim, o conceito de função é considerado como um conteúdo unificador em currículos de Matemática (STEELE; HILLEN; SMITH, 2013).

No entanto, as pesquisas que estudam o tema destacam especialmente as dificuldades do processo de ensino-aprendizagem desse conteúdo. Ao analisarmos o desenvolvimento histórico do conceito de função, podemos afirmar que o ensino e a aprendizagem do conceito de função na formação do professor são processos evolutivos trabalhosos e que tais dificuldades perpassam por todos os níveis de escolaridade.

A questão que emerge é que os próprios professores precisam compreender de forma abrangente e aprofundada o conceito de função, pois para ensinar é preciso aprender. Ball (1988, p.13) argumenta que: "os professores não podem ajudar as crianças a aprender coisas que eles mesmos não entendem. Mais sutil e menos compreendido, é o modo como o conhecimento dos professores moldam as oportunidades de aprendizagem dos alunos". No processo de ensino, as dificuldades do conteúdo de função, presentes em todos os níveis de escolaridade, são possivelmente decorrentes da 
formação do sujeito.

No ensino superior, nos Cursos de Licenciatura em Matemática, muitas vezes, o conceito de função é considerado como um conteúdo básico e, portanto, os professores consideram que os alunos já o compreendem e não aprofundam as discussões sobre o conceito, o que não os levam a superar as lacunas do conceito construído no processo da formação do indivíduo. E, dessa forma, como apontam Barufi (1999) e Rezende (2003), há um baixo nível de aprendizagem e um alto nível de reprovações na disciplina de Cálculo Diferencial e Integral I, que tem como base o conceito de função. Assim, a "crise do Cálculo" pode estar relacionada com uma mediana compreensão de conceitos básicos.

As dificuldades de compreensão do conceito de função não constituem apenas uma problemática da Educação Superior, muito menos somente da Educação Básica. Ao considerarmos o desenvolvimento histórico do conceito de função, podemos observar que as ideias sobre esse conceito foram sofrendo mudanças e a sua definição foi sendo aprimorada. Sierpinska (1992), em sua pesquisa sobre a dificuldade de compreensão do conceito de função, aprofunda-se nos estudos epistemológicos do conceito e considera que os obstáculos epistemológicos contribuem para uma imperfeita compreensão do conceito de função ao longo de sua formação.

Segundo a perspectiva epistemológica, o processo de ensino e aprendizagem do conceito de função no processo de formação do educando está nitidamente relacionado com os obstáculos. O filósofo Gaston Bachelard (1996) foi o primeiro a definir uma noção de obstáculos epistemológicos, apontando-os como uma luta mental para libertarse das ilusões e alcançar o conhecimento.

Bachelard aborda a importância do espírito científico e seus estágios de formação, apresentando a noção de obstáculo epistemológico, que passou a fazer parte das concepções epistemológicas discutidas na ciência. Para ele, os obstáculos epistemológicos são barreiras para a formação de um espírito científico, pois levam a estagnação no processo do pensamento. Além disso, Bachelard enfatiza a importância de detectar os obstáculos epistemológicos surgidos ao longo da construção da ciência, que foram omitidos ou desconhecidos pela história, destruindo o que foi insatisfatoriamente estabelecido e construindo novas ideias. No entanto, detectar os obstáculos 
epistemológicos na construção histórica do conceito de função é um trabalho complexo que deve ser realizado com detalhe, imparcialidade e com o estudo minucioso de cada cultura e época específica, estudando o trabalho de diversos matemáticos.

Sierpinska (1992) pesquisou os obstáculos epistemológicos ligados propriamente ao conceito de função. A autora relata que a maioria das dificuldades é consequência do processo de ensino e de aprendizagem do conceito, uma vez que os obstáculos são concretizados pelo professor. Esses obstáculos epistemológicos na educação exercem papel fundamental, possibilitando relacionar a epistemologia e a didática.

As ações educativas escolhidas para serem trabalhadas com um determinado conceito podem originar obstáculos e, em consequência, a construção de um conceito errôneo por parte dos alunos. Assim, as ações educativas podem ser cruciais para uma compreensão do conteúdo, já que a dificuldade na compreensão do conceito de função pode estar relacionada com a forma como ela é apresentada pelo professor. Sierpinska (1992, p. 28), afirma que "se o obstáculo não for apenas nosso ou de algumas outras pessoas, mas for mais generalizado, ou foi generalizado em alguma época ou em alguma cultura, então ele é conhecido como um obstáculo epistemológico" e defende que obstáculos epistemológicos acabavam facilitando o aparecimento de obstáculos didáticos.

Para Sierpinska, as dificuldades de aprendizagem do conceito de função são o bloqueio em associar as diferentes formas de representações da função como tabelas, gráficos, diagramas e fórmulas, dificuldade em entender o significado de variável, não compreensão das manipulações simbólicas como $f(x), x \rightarrow y$, sen $(x+t)$, entre outras.

Sierpinska (1992) relatou dezesseis obstáculos epistemológicos e dezenove ações importantes para a compreensão do conceito de função. Os obstáculos epistemológicos (OE) são: $\mathrm{OE}$ (f) - 1: a Matemática não se preocupa com problemas práticos; $\mathrm{OE}$ (f) - 2: (Uma Filosofia da Matemática). As técnicas computacionais utilizadas na produção de tabelas de relações numéricas não merecem ser objeto de estudo em Matemática; OE (f) 3: Tomar as dependências como fenômenos, focar em como as coisas mudam, ignorando o que muda; OE (f) - 4: (Esquema inconsciente do pensamento). Pensando em equação de termos e incógnitas para serem extraídos deles; OE (f) - 5: (Esquema inconsciente do pensamento). Quanto à ordem das variáveis como irrelevante; OE (f) - 6: (Uma atitude 
em relação ao conceito de número). Uma concepção heterogênea de número; OE (f) - 7: (Uma atitude em relação à noção de número) Uma filosofia pitagórica do número: tudo é número; $\mathrm{OE}$ (f) - 8: Leis da Física e funções em Matemática não têm nada em comum, pertencem a diferentes domínios de pensamento; OE (f) - 9: Proporção é um tipo privilegiado de relação; OE (f) - 10: Forte crença no poder das operações formais sobre a expressão algébrica; OE (f) - 11: Apenas as relações que podem ser descritas por fórmulas analíticas são dignas de receber o nome de funções; OE (f) - 12: (Uma concepção de definição) A definição é uma descrição de um objeto conhecido por sentidos ou visão clara. A definição não determina o objeto; em vez disso, o objeto determina a definição. Uma definição não é vinculativa logicamente; $\mathrm{OE}$ (f) - 13: (Concepção de função) As funções são sequências; OE (f) - 14: (Concepção de coordenadas) As coordenadas de um ponto são segmentos de reta (não números); OE (f) - 15: (Concepção do gráfico da função) A representação gráfica de uma função é um modelo geométrico da relação funcional. Não precisa ser fiel, pode conter pontos $(x, y)$ de modo que a função não seja definida em $x$; OE (f) - 16: (Uma concepção de variável) As mudanças de uma variável são mudanças no tempo.

Entretanto, Sierpinska (1992) também expõe as ações importantes para a compreensão do conceito de função como, por exemplo: U (f) - 4: Discriminação entre dois modos de matemática: um em termos de quantidades conhecidas e desconhecidas, o outro - em termos de quantidades variáveis e constantes; $U$ (f) - 5: Discriminação entre a variável independente e a variável dependente; U (f) - 9: Discriminação entre uma função e as ferramentas analíticas (algébricas) utilizadas para descrever essas leis; U (f) 12: Discriminação entre os conceitos de função e relação.

Assim, para que haja uma compreensão do conceito de função, ler a definição não é suficiente; é necessário que o professor utilize exemplos, contraexemplos e aplicabilidade, dando significado ao conceito abordado.

Nesta perspectiva, o estudo do conceito de função deve envolver diferentes representações, como tabelas, gráficos, diagramas, expressões algébricas (expressões analíticas ou fórmulas). As diferentes linguagens do conceito de função são fundamentais para o seu ensino e, portanto, devem ser abordadas ainda nos anos iniciais do Ensino 
Fundamental. Trindade e Moretti (2000) enfatizam a importância das diversas maneiras de representações de função como forma para superar os obstáculos epistemológicos de Sierpinska (1992). O uso de tabelas, por exemplo, em que existe uma relação funcional entre os elementos da linha de entrada correlacionados com os elementos da coluna de saída, é uma forma de trabalhar com os diferentes modos de representação de função possibilitando romper os obstáculos. Segundo Steele, Hillen e Smith (2013), o uso de tabelas propicia uma melhor perspectiva da particularidade do conceito de função, em que os dados que entram e saem da tabela podem possibilitar a compreensão quando duas grandezas são proporcionais ou não proporcionais. Sierpinska (1992) concorda com a importância da visualização da relação entre os pares ordenados $(x, y)$ por meio de tabelas e a construção de sequências numéricas e Lima (2008) afirma que o uso de tabelas pode auxiliar os professores na "visualização das regularidades numéricas, associando os resultados às representações gráficas, integrando, assim, os tipos de conhecimento de função." (LIMA, 2008, p. 54).

Assim como a tabela, o uso do diagrama para representar a relação entre os pares ordenados pode auxiliar o aluno a visualizar a transformação do elemento de um conjunto em outro elemento de outro conjunto e, também, associar a lei que define a função. Outro aspecto do conceito de função é compreender as variáveis e saber distinguir a dependência que há entre elas.

Ainda outro modo de comunicação por meio do uso de representações gráficas é uma linguagem do conceito de função que permite visualizar melhor o comportamento da função, o crescimento e os máximos e mínimos, continuidade e linearidade, entre outros. No gráfico, composto por pares ordenados $(x, y), x$ pertencerá ao conjunto domínio da função e $y$ pertencerá ao conjunto contradomínio da função ou ao subconjunto desse contradomínio denominado de imagem da função.

O conceito de função é apresentado, em geral, como expressão analítica, depois a partir da construção de uma tabela e, por fim, pela construção da representação gráfica. Consequentemente, os alunos têm a imagem do conceito de função apenas como uma expressão analítica e não percebem as outras como representação de função. Trindade e Moretti (2000) não desconsideram a importância das expressões algébricas, que por si só 
já são notórias, mas sugerem que se inicie a apresentação do conceito por meio de representações numéricas e gráficas, pois possibilitam uma melhor compreensão, "Tratase de primeiro desenvolver o conceito intuitivo de função, para depois formalizá-lo". (Trindade; Moretti, 2000, p. 44).

O conceito de função é fundamental, porém, para que os alunos possam compreender esse conceito, é preciso que, em seu ensino, haja uma organização nas representações de funções de acordo com os níveis de aprendizagem e o desenvolvimento de atividades apropriadas e sistematizadas para estruturar esse conceito.

\section{O CONHECIMENTO SOBRE O CONCEITO DE FUNÇÃO DE ALUNOS DO ÚLTIMO ANO DE UM CURSO DE LICENCIATURA EM MATEMÁTICA}

Aqui apresentamos uma análise e discussão dos dados obtidos por meio de um questionário aplicado aos alunos do último ano de um Curso de Licenciatura em Matemática de uma universidade pública do interior do Estado de São Paulo, com o intuito de investigar o conhecimento dos futuros professores de Matemática sobre o conceito de função. As atividades abordadas no questionário foram elaboradas considerando os obstáculos epistemológicos de Sierpinska (1992) em relação ao conceito de função.

A opção em aplicar o questionário para alunos do último ano do curso de Licenciatura em Matemática se deve ao fato de termos como objetivo discutir o conhecimento de futuros professores de Matemática e apontar suas possíveis implicações para a atividade docente sobre o ensino e a aprendizagem desse conceito. $\mathrm{O}$ questionário foi aplicado aos alunos do último ano do Curso que se voluntariaram em colaborar com a pesquisa. Esses alunos, que estavam no último ano do Curso, já haviam cursado a maioria das disciplinas da Estrutura Curricular que abordam o conceito de Função.

O questionário foi composto por nove questões elaboradas em conformidade com o objetivo da pesquisa e considerando alguns dos obstáculos apontados por Sierpinska (1992), ou seja, as dificuldades em relação ao conceito de função, como o bloqueio em associar as diferentes formas de representações de função (como, por exemplo: tabelas, gráficos, diagramas e fórmulas, por meio de conjuntos), dificuldade de entender o

ReviSeM, Ano 2019, N. 2, p. $48-70$ 
significado de variável e a não compreensão nas manipulações simbólicas como: $f(x)$, $x \rightarrow y, \operatorname{sen}(x+t)$. Desse modo, o questionário foi elaborado buscando explorar as diferentes representações de função.

Identificaremos os alunos que responderam ao questionário como: aluno 1, aluno 2 , aluno $3, \ldots$, aluno 15 . O questionário foi respondido por 15 alunos. Os alunos têm entre 20 e 29 anos, a maioria é do sexo feminino (12 alunas) e 2 alunas já lecionavam.

Para uma análise do questionário, inicialmente, foi feito um estudo geral dos erros dos alunos (tabela 1). Em seguida, passamos a analisar as justificativas e os procedimentos que levaram os alunos a escolher a alternativa dada como resposta.

Quadro 1 - Percentual de erros
\begin{tabular}{|l|l|l|l|l|l|l|l|l|l|}
\hline Questões & Q1 & Q2 & Q3 & Q4 & Q5 & Q6 & Q7 & Q8 & Q9 \\
\hline Erros & $14 \%$ & $14 \%$ & $20 \%$ & $20 \%$ & $67 \%$ & $40 \%$ & $34 \%$ & $0 \%$ & $27 \%$ \\
\hline
\end{tabular}

Fonte: Próprio autor

A tabela mostra que os alunos tiveram mais dificuldades nas questões 5, 6, 7 e 9 . As questões do questionário abordavam o conceito de função de diferentes formas: representação de função por tabelas (Questão 1), representação de função por diagramas (Questão 2), relação de variável dependente e independente dentro de uma situação problema (Questão 3), definição de função por meio de conjuntos (Questões 4 e 8), representação gráfica de função no plano cartesiano (Questões 5, 6 e 7) e notação simbólica para representar função (Questões 4, 8 e 9).

A primeira questão do questionário aborda a representação mediante tabelas, a segunda questão é apresentada por meio do uso de diagramas, a terceira teve o intuito de analisar a variação (variáveis independentes e dependentes), a quarta questão aborda a representação do conceito de função por meio da linguagem matemática, as questões cinco e seis utilizam a representação gráfica para abordar a definição de função, a questão sete também utiliza a representação gráfica, porém para tratar do domínio e do contradomínio da função e as questões oito e nove apresentam a formalização do conceito de função, do ponto de vista da notação. 
$1^{\circ}$ Questão: Os dados das tabelas abaixo poderiam representar uma função? Justifique sua resposta.

a)

\begin{tabular}{|c|c|c|c|c|c|}
\hline$X$ & 0 & 1 & 2 & 3 & 4 \\
\hline$Y$ & 5 & 9 & 13 & 17 & 21 \\
\hline
\end{tabular}

b)

\begin{tabular}{|c|c|c|c|c|c|c|c|}
\hline$X$ & 0 & -1 & 1 & -2 & 2 & 3 & -3 \\
\hline$Y$ & 0 & 1 & 1 & 4 & 4 & 9 & 9 \\
\hline
\end{tabular}

A primeira questão teve como propósito avaliar o nível de conhecimento dos alunos sobre a representação do conceito de função por meio de tabelas. Uma tabela é uma lista dos valores que a função assume para determinados valores da variável independente. Foram elaboradas duas tabelas e solicitado que fosse identificado se elas poderiam representar uma função. Dessa forma, esperava-se que os alunos percebessem a relação funcional correspondente entre as linhas e as colunas dessas tabelas. Em geral, o primeiro contato que os alunos do Ensino Básico têm com o conceito de função é por intermédio da representação por tabela.

Nesta questão apenas $14 \%$ dos alunos erraram a resposta ou não souberam responder. Na alternativa (a) os alunos não tiveram dificuldade em perceber a relação funcional entre os números das linhas e das colunas, por se tratar de uma função afim, ou seja, definida por $f(x)=4 x+5$, o que permitiu uma visualização mais clara de correspondência, transformação, dependência (uma grandeza em função de outra). Já na alternativa (b), por se tratar de uma função quadrática, em alguns casos, os alunos se confundiram ao tentar relacionar a definição de função com os valores apresentados na tabela. Assim, segundo Sierpinska (1992), trata-se de um obstáculo epistemológico em que o inconsciente do pensamento acredita que uma função somente se apresenta quando se consegue extrair incógnitas e equações de termos - OE (f) - 4 e OE (f) - 11. Em um dos questionários, o aluno respondeu que os dados (valores numéricos) presentes na tabela não poderiam representar uma função, uma vez que existiam valores distintos de $x$ com imagens iguais. O aluno, ao justificar a resposta ao item (b), parece ter se

ReviSeM, Ano 2019, N. 2, p. $48-70$ 57 
equivocado quando se lembrou da noção ou da definição de função - Para todo elemento $x$ do domínio existe um único elemento $y$ do contradomínio tal que o par ordenado $(x, y) \in f-$ não compreendendo a definição. No entanto, ao responder à questão 2 - com diagramas - o mesmo aluno marcou como correta uma alternativa em que todo elemento do domínio está relacionado a um único elemento do contradomínio. Ou seja, ele não compreendeu devidamente o conceito de função em diferentes representações.

A representação de função mediante tabelas surgiu historicamente muito antes da definição formalizada do conceito. Porém, a sequência didática para o ensino de funções, usualmente apresentada nos livros e seguida pelos professores, faz o caminho inverso, ou seja, a representação de função por meio de tabelas é apresentada após a formalização do conceito. Segundo Trindade e Moretti (2000), a apresentação do conceito de função é desenvolvida, em um primeiro momento, pela representação simbólica, em seguida, pela construção de tabelas e, por fim, por meio de representação gráfica.

Dos 15 questionários respondidos, 13 alunos, além de responder o que foi solicitado na questão, também encontraram as expressões algébricas que representavam as funções. De fato, segundo Sierpinska (1992), parece haver uma ênfase na abordagem sobre as expressões algébricas, pois ao analisar a representação gráfica ou a tabela de valores para encontrar a resposta, o aluno escreve a lei da função, ou seja, há uma forte associação, por parte dos alunos, entre o conceito de função e as relações que podem ser descritas por fórmulas analíticas.

Assim como a representação tabular da função, a representação em diagrama pode auxiliar o aluno a visualizar a transformação entre um elemento de um conjunto em outro elemento de outro conjunto e, além disso, associar a relação funcional que define a função. A escolha da segunda questão foi proposital, pelo fato de que a representação em diagrama exerce uma linguagem compreensível do movimento aparente do conceito de função, o que auxilia em detectar se o diagrama representa função ou não, além de que no Ensino Básico a definição de função é abordada juntamente com a forma de diagrama. Vemos que $86 \%$ dos alunos acertaram a resposta. 
$2^{\circ}$ Questão: Quais dos seguintes diagramas representam uma função $f$ de $A$ em $B$ ?

a)

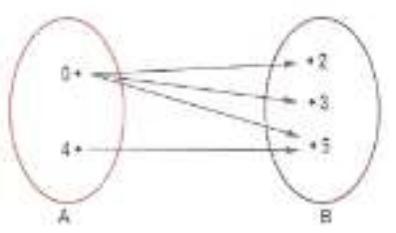

c)

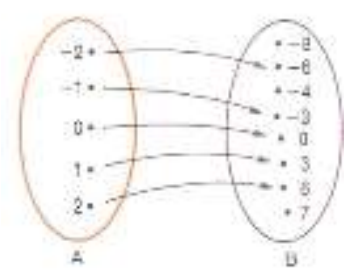

b)

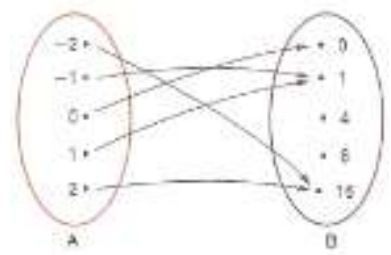

d)

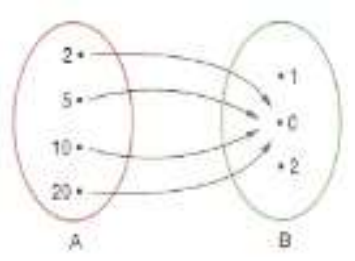

Parece haver uma melhor compreensão do conceito de função quando a representação utilizada é o diagrama, já que os 2 alunos que erraram a primeira questão acertaram a segunda. Com isso, percebemos um obstáculo entre a representação de função por meio de tabela e de diagrama. Nesse sentido, podemos conjecturar que o uso do diagrama para representar uma função é mais compreendido pelos alunos do que as outras representações. Apesar de parecer uma questão simples, nem todos os alunos acertaram a resposta. A alternativa (c) foi a única que não trouxe dúvidas, talvez em razão da esquematização linear (setas). Já as alternativas que também são corretas, alternativa (b) e alternativa (d), trouxeram dúvidas para alguns alunos.

A representação mediante diagramas geralmente é abordada concomitante com a expressão analítica, pois de forma intuitiva, auxilia na compreensão da relação funcional ou "lei de formação", ou melhor, instrui como obter o valor $f(x) \in B$ quando é fornecido $x \in A$, com o propósito que a função $f$ tenha o conjunto $A$ como seu domínio e a "lei de formação" produza o valor $f(x) \in B$ para todo elemento do conjunto $A$ e, ainda, para cada elemento do conjunto $A$ do domínio da função $f$ a "lei de formação" deve fazer corresponder um único elemento $f(x)$ do conjunto $B$ do contradomínio da função $f$. E, quando é apresentada dessa forma, a esquematização linear (setas) auxilia na identificação se o diagrama representa uma função ou não, facilitando também 
determinar o domínio, contradomínio e a imagem da função.

Observe que para termos uma função, todos os elementos do conjunto $A$ devem possuir esquematização linear (setas) em direção a um único elemento do conjunto $B$.

Percebemos que há uma utilização excessiva, no uso de diagramas para representar a relação das funções, de números inteiros. Isso pode levar o aluno, quando ele começar a estudar a representação gráfica de funções, a não encontrar conexão entre essa representação e a representação com diagramas, já que, na representação gráfica, passa-se a utilizar números reais. Além disso, ao comparar tais representações, o aluno poderia compreender a diferença entre conjunto discreto e conjunto contínuo.

$3^{\text {o }}$ Questão: A distância $d$ (em $\mathrm{km}$ ) que um carro viaja em $t$ horas a $75 \mathrm{~km} / \mathrm{h}$ é $d=$ 75t. Qual a variável dependente? Qual a variável independente? Por quê?

A terceira questão teve como objetivo compreender a relação de dependência que as duas questões anteriores trabalharam de forma implícita - e avaliar se o aluno tem uma visão clara da dependência funcional (variável dependente) que se apresenta no conceito de função. Abordamos na questão um problema prático que envolve o entendimento de dependência funcional e variável independente. Segundo Cotret (1988),

É interessante notar que, nas primeiras definições do conceito de função, as noções centrais são variação e dependência; a correspondência está presente, mas de forma implícita. Então, quanto mais nos aproximamos das definições modernas, o desaparecimento mais gradual da variação, então a dependência, que podemos encontrar alguns traços com a regra de correspondência, levando eventualmente a uma correspondência pura. (COTRET, 1988, p. 8).

A conexão da definição matemática com problemas do "mundo" é um dos quesitos do próprio conceito de função, pois matematicamente uma função $f: A \rightarrow B$, cujo conjunto A denomina-se o domínio de $f$, cujo $x$ um elemento de $A$ é designado como a variável independente ou argumento da função $f$, ao passo que o elemento genérico $y=f(x)$ do conjunto $B$, no qual a função $f$ toma valores, denomina-se contradomínio de $f$, segundo elemento dos pares ordenados $(x, y) \in f$, tal que $y$ é denominado variável dependente da função $f$. O elemento dependente vai variar de acordo com o elemento do qual ele depende, ou seja, o elemento independente. Assim, a distância $d$ depende do tempo $t$. 
O percentual de erros da questão foi de 14\%, que segundo Sierpinska (1992), consiste no primeiro obstáculo, ou seja, há uma dificuldade de se reconhecer a presença da Matemática em uma atividade do cotidiano. Outro obstáculo envolvido na resolução da questão é o oitavo, no qual Matemática e Física não têm nada em comum, pertencendo a diferentes domínios de pensamento. Observemos a Figura 1, em que o Aluno 1 encontra dificuldades em detectar a variável que depende, pois, o tempo é uma variável independente o que está dependendo é a distância.

\section{A distância $d(\mathrm{em} \mathrm{km})$ que um carro viaja em $t$ horas a $75 \mathrm{~km} / \mathrm{h}$ é $d=75 t$. Qual a} variável dependente? Qual a variável independente? Por quê?

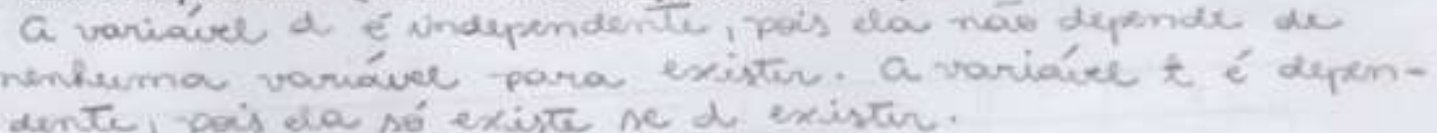

Figura 1 - Questionário do Aluno 1

Fonte: Elaborado pelo autor

Muitas vezes, a confusão feita entre variável dependente e independente pode estar também ligada ao terceiro obstáculo epistemológico de Sierpinska (1992), em que se tomam as dependências como fenômenos, focando em como as coisas mudam e ignorando o que muda.

As leis físicas estabelecem-se mediante a generalização de dados obtidos por meio de experimentos e sua veracidade comprova-se quando há uma correspondência entre as conclusões com a prática. Assim, as leis físicas expressam a relação interna e objetiva entre os fenômenos físicos e a dependência real entre as grandezas físicas. Nesse sentido, os conteúdos das leis físicas expressam-se matematicamente como uma dependência dos valores numéricos $\alpha$ e $\beta$ das grandezas físicas dadas A e B. Nesta perspectiva, o professor pode trabalhar com situações do cotidiano e criar problemas cuja solução envolva a compreensão das relações de dependência e de independência presentes nas leis físicas. No entanto, os problemas do cotidiano são intuitivos e não abordam as fórmulas, talvez isso gere dúvida para o aluno, pois a definição de função é dada de forma "pronta", sem estimular o aluno a pensar no conceito de variação, podendo surgir uma barreira entre fórmulas e os conceitos envolvidos de dependência e independência.

Assim, entendemos que alguns alunos ainda têm dificuldades em detectar as 61

ReviSeM, Ano 2019, N. 2, p. $48-70$ 
variáveis em situações que envolvem fenômenos da natureza e ter um olhar matemático para um problema da Física ou outras áreas do conhecimento.

Mediante essa análise e na sequência em que o conceito de função é apresentado, percebe-se que o conceito é usado como objeto de estudo e não como ferramenta para resolver problemas, que sejam empíricos ou não.

$4^{\text {o }}$ Questão: Sejam $A$ e $B$ conjuntos. Dizemos que $f: A \rightarrow B$ é uma função de $A$ em $B$ quando:

a) Para todo $x \in A, \exists y \in B ; f(x)=y$. ( ) Verdadeira ( ) Falsa

b) Se a relação $f: X \rightarrow Y$ é uma função, é verdade que: $x_{1}, x_{2} \in X$, $x_{1} \neq x_{2} \Rightarrow f\left(x_{1}\right) \neq f\left(x_{2}\right)$. ( ) Verdadeira ( ) Falsa

c) A relação $f: X \rightarrow Y$ é uma função se para $\mathrm{y} \in \mathrm{B}$, existe um $\mathrm{x} \in \mathrm{A}$ tal que $\mathrm{f}(\mathrm{x})=\mathrm{y}$. ( ) Verdadeira ( ) Falsa

d) A relação $f: X \rightarrow Y$ é uma função se dados $x_{1}, x_{2} \in X$, em que $x_{1}=x_{2}$, temos $f\left(x_{1}\right)=f\left(x_{2}\right)$. ( ) Verdadeira ( ) Falsa

A quarta questão teve o objetivo de avaliar a compreensão da linguagem em símbolos da Matemática, de maneira a reconhecer uma função por meios da escrita matemática. Assim, diz-se que uma função $f: X \rightarrow Y$ é sobrejetora quando para todo elemento $y$ de $Y$ existe ao menos um elemento $x$ de $X$ tal que $f(x)=y$. Em outras palavras, uma função $f$ de $X$ em $Y$ é sobrejetora quando a sua imagem $f(X)$ coincide com seu contradomínio $Y$, isto é, $f(X)=Y$. Ou ainda, quando uma função $f: X \rightarrow Y$ é sobrejetora também significa que $f$ é uma sobrejeção de $X$ em $Y$ ou que $f$ é uma função de $X$ sobre $Y$. Desse modo, em símbolos, essa qualidade da função, exprime-se da seguinte forma: $f: X \rightarrow Y$ é sobrejetora $\Leftrightarrow \forall y \in Y, \exists x \in X: y=f(x)$.

E, que uma função $f: X \rightarrow Y$ é injetora quando dois elementos distintos quaisquer $x_{1}$ e $x_{2}$ de $X$ têm sempre imagens pela função $f$ também distintas $f\left(x_{1}\right)$ e $f\left(x_{2}\right)$. Em outras palavras, uma função injetora $f: X \rightarrow Y$ transforma elementos distintos do conjunto $X$ em elementos também distintos do conjunto $Y$. Assim, em símbolos, essa qualidade da função, exprime-se da seguinte forma: $f: X \rightarrow Y$ é injetora $\Leftrightarrow\left(\forall x_{1}, x_{2} \in X \wedge x_{1} \neq x_{2}\right) \Rightarrow$ $f\left(x_{1}\right) \neq f\left(x_{2}\right)$, ou seja, de forma equivalente: $f: X \rightarrow Y$ é injetora $\Leftrightarrow\left(\forall x_{1}, x_{2} \in X \wedge\right.$ 
$\left.f\left(x_{1}\right)=f\left(x_{2}\right)\right) \Rightarrow x_{1}=x_{2}$.

$\mathrm{Na}$ questão 4 houve um percentual de $80 \%$ dos alunos que julgaram a alternativa a) verdadeira, porém, nas outras alternativas consideradas falsas, nem todos os alunos apresentaram um argumento para sua escolha. Na alternativa b) 12 alunos responderam que era falsa, 4 alunos que era verdadeira e 1 aluno não soube responder. Já na alternativa c), que corresponde a definição de função sobrejetora, 7 alunos responderam como falsa, 4 alunos responderam verdadeira e 4 alunos não souberam responder. Na alternativa d) 10 alunos julgaram como verdadeira e 4 alunos como falsa, 1 não respondeu.

O desempenho dos alunos na questão mostra certa dificuldade em relação à definição formal do conceito de função que utiliza uma linguagem matemática simbólica, gerando dúvidas quanto a definição de função injetora ou sobrejetora. A interpretação da linguagem matemática por meio dos símbolos é enfatizada no ensino do conceito de função e, desse modo, os alunos não conseguem relacionar a expressão analítica de uma função com as outras representações, ou seja, acreditam que se a função não é dada por uma "expressão analítica", então, não é uma função. Esse aspecto reforça o obstáculo epistemológico da concepção de que a definição é a forma correta e única de representar uma função. $\mathrm{OE}(\mathrm{f})$ - 10: A forte crença no poder das operações formais como expressões algébricas.

$5^{0}$ Questão: Quais das relações de $\mathbb{R}$ em $\mathbb{R}$, cujos gráficos aparecem abaixo, representam funções? Justifique sua resposta.
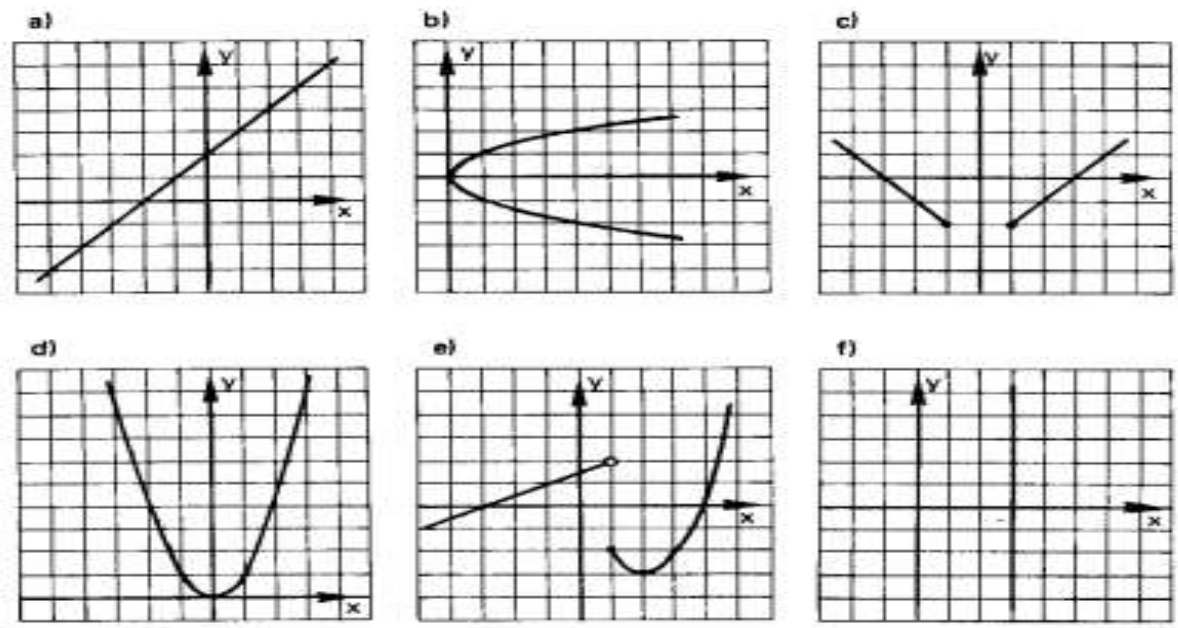
A quinta questão foi a que teve o maior número de erros. Mais de $60 \%$ dos alunos erraram a questão, não a responderam ou deixaram-na incompleta. Com o objetivo de trazer diferentes formas de se representar o conceito de função, a quinta questão abordou as representações gráficas. Assim, nessa situação, foi usado o plano cartesiano para representar função. O estudo das representações gráficas, segundo Trindade e Moretti (2000), é fundamental para a compreensão do conceito de função. Os autores enfatizam que o uso da representação gráfica de função é o melhor meio para se trabalhar a linearidade, os intervalos de crescimento e decrescimento, os máximos e mínimos, a taxa de variação, regularidade e a continuidade.

O gráfico da função $f: X \rightarrow Y$ é o subconjunto $G$ do produto cartesiano $X \times Y$ formado pelos pares ordenados relacionados mediante uma correspondência entre os elementos do conjunto domínio e do conjunto imagem. Assim, em símbolos, exprime-se da seguinte forma: $G=\{(x, y) \in X \times Y: y=f(x)\}=\{(x, f(x): x \in X)\}$. Assim, esse conjunto é denominado de gráfico de $f$ porque permite estabelecer uma representação gráfica no caso em que $X$ e $Y$ são conjuntos de números reais. Na prática, podem-se representar os elementos dos conjuntos $X$ e $Y$ em dois eixos ortogonais, cada par ordenado de elementos de $G$ pode ser representado por um ponto no plano cartesiano referente a esses dois eixos.

O cuidado no ensino da representação gráfica de uma função é importante para uma compreensão ampla do conceito, pois, muitas vezes, até nos livros didáticos os gráficos são apresentados sem escalas e sem papel quadriculado. Dessa forma, a construção do gráfico sem escala ou sem o uso de papel milimetrado pode causar uma distorção do gráfico e, consequentemente, torná-lo apenas um modelo da relação funcional, mas que não a representa, constituindo um obstáculo epistemológico (OE(f) 15).

$\mathrm{Na}$ questão proposta, foram apresentadas seis representações gráficas e foi solicitado que os alunos determinassem quais representavam funções. Ao analisar as respostas, uma que chamou a atenção foi a do Aluno 1 que respondeu que todas as representações são funções, sem compreender que a representação gráfica apresentada no

ReviSeM, Ano 2019, N. 2, p. $48-70$ 64 
item (b) é um contraexemplo claro para a definição de função.

Nessa questão, as respostas foram variadas e as representações gráficas (c) e (e) geraram mais dúvida do que a (b). O fato de aparecerem intervalos abertos e fechados nos itens (c) e (e) podem ter motivado os alunos a marcarem essas alternativas como não sendo a representação gráfica de uma função. Para identificar se uma curva representa ou não o gráfico de uma função, podemos traçar retas paralelas ao eixo de ordenada $y$ do sistema de coordenadas cartesianas e verificar quantas vezes essas retas intersectam a curva representada pelo gráfico no plano cartesiano: se intersectam mais de uma vez a curva, então não se trata de gráfico de função, contrapondo a definição. Além disso, podemos perceber que os alunos não tiveram dificuldade em enunciar a definição de função formal usando a simbologia matemática nas questões 8 e 9 seguintes, no entanto ao impormos questões que apresentam diferentes representações, houve conflitos.

$6^{\mathbf{0}}$ Questão: Com relação ao gráfico abaixo, justifique a afirmação que julgar falsa ou verdadeira.

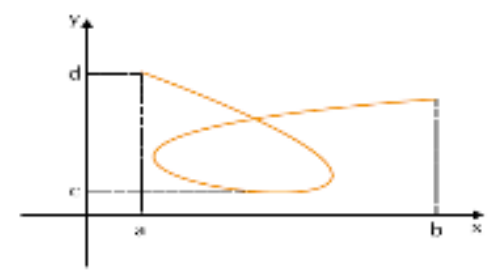

1. $\quad$ Representa uma função $f:[a, b] \rightarrow \mathbb{R}$. Verdade ( ) Falsa ( )

2. Não representa uma função de $[a, b]$ em $\mathbb{R}$ porque existe $y \in \mathbb{R}$ que não é imagem de qualquer $x \in[a, b]$. Verdade ( ) Falsa ( )

3. Não representa uma função de $[a, b]$ em $\mathbb{R}$ porque existe elemento $x \in[a, b]$, com mais de uma imagem. Verdade ( ) Falsa ()

4. Representa uma função $f:[a, b] \rightarrow[c, d]$. Verdade ( ) Falsa ( )

5. Representa uma função bijetora. Verdade ( ) Falsa ( )

A questão de número seis faz uma conexão entre a representação gráfica e a linguagem matemática de uma função. Nas respostas dadas, observamos que os alunos fizeram confusão com as alternativas apresentadas. 
$7^{0}$ Questão: Os seguintes gráficos representam funções; determine o domínio $D$ e o conjunto imagem Im de cada um deles:
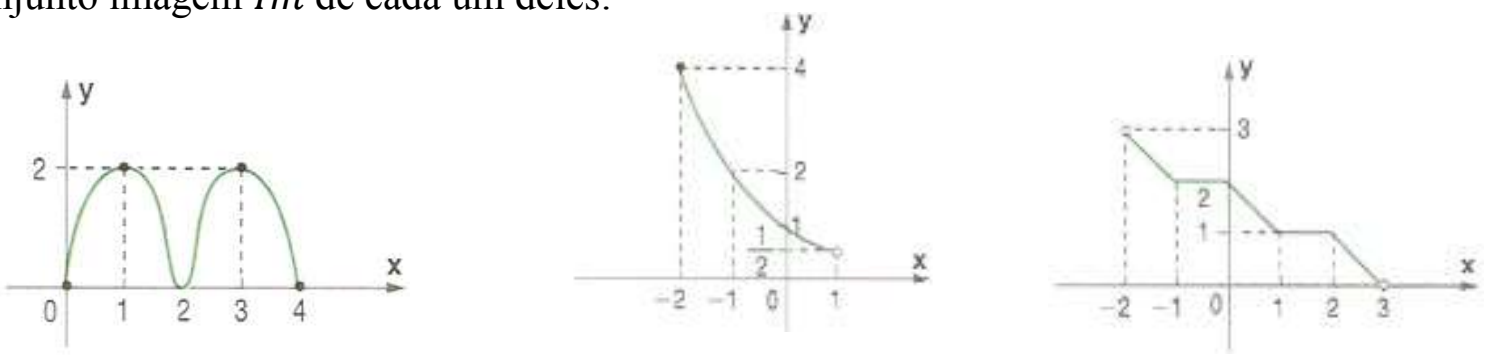

O objetivo da sétima questão era avaliar a compreensão da linguagem gráfica, ou seja, analisar os intervalos em que a função está compreendida. Para a resolução da questão era necessário determinar o domínio e a imagem de cada função.

Examinar o gráfico pode nos fornecer várias informações sobre uma dada função, mesmo sem conhecermos a expressão algébrica da função.

$\mathbf{8}^{\mathbf{0}}$ Questão: Considere a notação $f: A \rightarrow B$. O que significa essa notação? Explique cada componente dessa notação.

$9^{0}$ Questão: Agora, considere a notação $x \mapsto f(x)$. O que significa essa notação? Explique cada componente dessa notação.

As escolhas das questões oito e nove tiveram como propósito analisar a compreensão dos alunos sobre as notações muito usadas nos livros, e se esperava o reconhecimento de cada uma delas. A compreensão da notação de função parece não ser uma dificuldade dos alunos, tendo em vista as respostas obtidas por meio do questionário. Pelo contrário, percebemos uma habilidade por parte dos alunos em compreender as notações usadas, provavelmente, em razão da ênfase dada a esse aspecto no decorrer do curso. Entretanto, não podemos afirmar que a compreensão da notação implica na compreensão do conceito, pois percebemos dificuldades nas questões anteriores por parte de alguns alunos.

A maioria dos alunos (70\%) soube responder de forma satisfatória as questões $8 \mathrm{e}$ 9. O conceito de função representado por expressão matemática de uma função em que $f$ associa cada ponto $x$ de um conjunto $A$ a um ponto ou valor $f(x)$ de um conjunto $B$ é dada por: $f: A \rightarrow B, x \mapsto f(x)$. Assim, $A$ é o domínio da $f$ e o contradomínio é $B$. Nesta

ReviSeM, Ano 2019, N. 2, p. $48-70$ 66 
situação, observamos nos questionário que essas notações são compreendidas pelos alunos.

De modo geral, observamos que os alunos tiveram mais dificuldades nas questões que envolviam a representação de função por gráficos e tabela e na questão sobre a definição de função. Por outro lado, os alunos tiveram facilidade nas questões que envolviam notação e diagramas. Tais resultados podem estar relacionados às ênfases que são dadas ao estudo das funções na graduação.

Outro ponto que destacamos é a associação que os alunos fazem entre "função" e “expressão algébrica para representar uma função". Essa associação leva os alunos a não considerarem como uma função os exemplos em que a expressão algébrica não é única ou em que a função não pode ser representada algebricamente.

\section{CONSIDERAÇÕES}

A nossa análise das respostas dadas pelos alunos do $4^{\circ}$ ano do Curso de Licenciatura em Matemática mostra que eles tiveram um bom desempenho, porém que alguns obstáculos epistemológicos necessitam de atenção. Podemos afirmar que alguns alunos acreditam que uma função deve envolver incógnitas e equações de termos, conforme os obstáculos OE (f) - 4 e OE (f) - 11, apontados por Sierpinska (1992); há também uma dificuldade de se reconhecer a presença da Matemática em uma atividade do cotidiano. Outro obstáculo envolvido é a visão de que Matemática e Física não têm nada em comum. Também parece haver uma ênfase na abordagem sobre as expressões algébricas, pois ao analisar a representação gráfica ou a tabela de valores para encontrar uma resposta a uma dada questão, há alunos que escrevem a lei da função antes de apontar uma resposta, ou seja, há uma forte associação, por parte dos alunos, entre o conceito de função e as relações que podem ser descritas por fórmulas analíticas. Outro obstáculo é tomar dependências como fenômenos, focando em como as coisas mudam e ignorando o que muda.

Também notamos que a interpretação da linguagem matemática por meio dos símbolos parece ser enfatizada no ensino do conceito de função e, desse modo, os alunos não conseguem relacionar a expressão analítica de uma função com as outras

ReviSeM, Ano 2019, N. 2, p. $48-70$ 67 
representações, ou seja, acreditam que se a função não é dada por uma "expressão analítica", então, não é uma função. Esse aspecto reforça o obstáculo epistemológico da concepção de que a definição é a forma correta e única de representar uma função. Tais resultados podem estar relacionados às ênfases que são dadas ao estudo das funções na graduação, que em síntese abordam significantemente o uso de expressões analíticas como representação de função, e desviam a importância dos estudos das demais representações de função.

O conceito de função apresenta em seu ensino "obstáculos epistemológicos" que precisam ser superados. Pelos resultados obtidos em nossa pesquisa, percebemos que os obstáculos estão presentes e não foram totalmente superados, mesmo por alunos do último ano do curso.

Diante dos resultados obtidos, podemos apontar quatro implicações dos conhecimentos dos futuros professores de Matemática sobre função para a atividade docente: a primeira é que é provável que, em sua prática docente, ao abordar o conceito de função, o professor enfatize exemplos de função que envolvam incógnitas e equações de termos; a segunda é a dificuldade de desenvolver estratégias de ensino que mostrem a presença da Matemática em atividades do cotidiano; a terceira é a dificuldade em promover um ensino de Matemática interdisciplinar com outras áreas de conhecimento, como, por exemplo, com a Física; a quarta é a possibilidade de haver uma ênfase no trabalho com expressões algébricas, reforçando nos alunos uma ideia errônea de associação entre o conceito de função e as relações que podem ser descritas por fórmulas analíticas, em detrimento de um trabalho que envolva também, por exemplo, representação gráfica ou tabela de valores.

Conforme atesta a história, o conceito de função é fundamental para a organização dos ramos e dos conhecimentos específicos da Matemática, desempenhando também um papel importante em outras áreas do conhecimento, em virtude de sua aplicabilidade. De fato, o conceito de função está presente no currículo da Educação Básica, em nível fundamental e médio, bem como em diferentes cursos de graduação. Em especial, no Curso de Licenciatura em Matemática o conceito de função é fundamental para a compreensão de muitos conceitos e para a formação do professor de Matemática. 
Cintra, F. P.; Balieiro Filho, I. F.

\section{REFERÊNCIAS}

BACHELARD, G. A formação do espírito científico: contribuição para uma psicanálise do conhecimento. Rio de Janeiro: Contraponto, 1996.

BALL, D. L. Research on teaching mathematics: making subject-matter knowledge part of the equation. In: BROPHY, J. (Ed.). Advances in research on teaching. Greenwich: JAI Press, 1988.

BARUFI, B. C. M. A construção/negociação de significados no curso universitário inicial de Calculo Diferencial e Integral. 1999. 184 f. Tese (Doutorado em Educação) Faculdade de Educação, Universidade de São Paulo, São Paulo, 1999.

COTRET, S. R. Une experimentation sur les conceptions de la notion de fonction a travers les representations graphiques du mouvement. In: SEMINAIRE DE DIDACTIQUE DES MATHÉMATIQUES ET DE L'INFORMATIQUE. Année 19861987, Année 1987-1988, Grenoble. LSD- IMAG. p. 115-138.

LIMA, L. A aprendizagem significativa do conceito de função na formação inicial do professor de matemática. 2008. 157 f. Dissertação (Mestrado em Educação) Universidade Estadual do Ceará, Fortaleza, 2008.

LLINARES, S. Conocimiento profesional del profesor de matemáticas: conocimiento, creencias y contexto en relación a la noción de función. En J. P. Ponte, C. Monteiro, M. Maia, L. Serrazina y C. Loureiro (Ed.), Desenvolvimento Profissional dos professores de Matemática. Que Formação? (pp. 47-82). Lisboa: Sociedade Portuguesa de Ciências da Educação, 1996.

REZENDE, W. M. O Ensino de cálculo: dificuldades de natureza epistemológica. 2003. 468 f. Tese (Doutorado em Educação) - Faculdade de Educação, Universidade de São Paulo, São Paulo, 2003.

SIERPINSKA, A. On understanding the notion of function. Em: DUBINSKY, E; HAREL, G. (Ed.) The Concept of Function: aspects of epistemology and pedagogics. Washington: Mathematical Association of America, 1992.

SHULMAN, L. Those who understand: knowledge growth in teaching. Educational Researcher, v. 15, n. 2, p. 4-14, 1986.

STEELE, M.; HILLEN, A. F.; SMITH, M. S. Developing mathematical knowledge for teaching in a methods course: the case of function. Journal of Mathematics Teacher Education, [S. 1.], v. 16, I. 6, p. 451-483. 2013.

TRINDADE, J. A. de; MORETTI, M. T. Uma relação entre a teoria histórico cultural e a

ReviSeM, Ano 2019, N. 2, p. $48-70$ 
epistemologia histórico-crítica no ensino de funções: a mediação. Revista Zetetiké, CEPEMFE/UNICAMP, n. 13-14, p. 29-49, 2000.

Submetido em 02 de setembro de 2019. Aprovado em 21 de outubro de 2019. 